\title{
A Framework to Synergize Partial Order Reduction with State Interpolation
}

\author{
Duc-Hiep Chu and Joxan Jaffar \\ National University of Singapore \\ hiepcd, joxan@comp.nus.edu.sg
}

\begin{abstract}
We address the problem of reasoning about interleavings in safety verification of concurrent programs. In the literature, there are two prominent techniques for pruning the search space. First, there are wellinvestigated trace-based methods, collectively known as "Partial Order Reduction (POR)", which operate by weakening the concept of a trace by abstracting the total order of its transitions into a partial order. Second, there is state-based interpolation where a collection of formulas can be generalized by taking into account the property to be verified. Our main contribution is a framework that synergistically combines POR with state interpolation so that the sum is more than its parts.
\end{abstract}

\section{Introduction}

We consider the state explosion problem in safety verification of concurrent programs. This is caused by the interleavings of transitions from different processes. In explicit-state model checking, a general approach to counter this explosion is Partial Order Reduction (POR) (e.g., $[20,18,9])$. This exploits the equivalence of interleavings of "independent" transitions: two transitions are independent if their consecutive occurrences in a trace can be swapped without changing the final state. In other words, POR-related methods prune away redundant process interleavings in a sense that, for each Mazurkiewicz $[16]^{1}$ trace equivalence class of interleavings, if a representative has been checked, the remaining ones are regarded as redundant.

On the other hand, symbolic execution [15] is another method for program reasoning which recently has made increasing impact on software engineering research [4]. The main challenge for symbolic execution is the exponential number of symbolic paths. The works $[17,12]$ tackle successfully this fundamental problem by eliminating from the concrete model, on-the-fly, those facts which are irrelevant or too-specific for proving the unreachability of the error nodes. This learning phase consists of computing state-based interpolants in a similar spirit to that of conflict clause learning in SAT solvers.

Now symbolic execution with state interpolation (SI) has been shown to be effective for verifying sequential programs. In SI [17,12], a node at program

\footnotetext{
${ }^{1}$ We remark that the concept of POR goes beyond the preservation of Mazurkiewicz traces, e.g. [20]. However, from a practical perspective, it is safe to consider such form of pruning as a representative example of POR.
} 
point $\ell$ in the reachability tree can be pruned, if its context is subsumed by the interpolant computed earlier for the same program point $\ell$. Therefore, even in the best case scenario, the number of states explored by an SI method must still be at least the number of all distinct program points ${ }^{2}$. However, in the setting of concurrent programs, exploring each distinct global program point ${ }^{3}$ once might already be considered prohibitive. In short, symbolic execution with SI alone is not efficient enough for the verification of concurrent programs.

Recent work (e.g., [25]) has shown the usefulness of going stateful in implementing a POR method. It directly follows that SI can help to yield even better performance. In order to implement an efficient stateful algorithm, we are required to come up with an abstraction for each (concrete or symbolic) state. Unsurprisingly, SI often offers us good abstractions.

The above suggests that POR and SI can be very much complementary to each other. In this paper, we propose a general framework employing symbolic execution in the exploration of the state space, while both POR and SI are exploited for pruning. SI and POR are combined synergistically as the concept of interpolation. Interpolation is essentially a form of learning where the completed search of a safe subtree is then formulated as a recipe, ideally a succinct formula, for future pruning. The key distinction of our interpolation framework is that each recipe discovered by a node is forced to be conveyed back to its ancestors, which gives rise to pruning of larger subtrees.

In summary, we address the challenge: "combining classic POR methods with symbolic technique has proven to be difficult" [14], especially in the context of software verification. More specifically, we propose an algorithm schema to combine synergistically POR with state interpolation so that the sum is more than its parts. However, we first need to formalize POR wrt. a symbolic search framework with abstraction in such a way that: (1) POR can be property driven and (2) POR, or more precisely, the concept of persistent set, can be applicable for a set of states (rather than an individual state). While the main contribution is a theoretical framework (for space reason, we present the proofs of the two theorems in the Appendix), we also indicate a potential for the development of advanced implementations.

\section{Related Work}

Partial Order Reduction (POR) is a well-investigated technique in model checking of concurrent systems. Some notable early works are [20,18,9]. Later refinements of POR, Dynamic [8] and Cartesian [11] POR (DPOR and CPOR respectively) improve traditional POR techniques by detecting collisions on-the-fly. Recently, [1]

\footnotetext{
${ }^{2}$ Whereas POR-related methods do not suffer from this. Here we assume that the input concurrent program has already been preprocessed (e.g., by static slicing to remove irrelevant transitions, or by static block encodings) to reduce the size of the transition system for each process.

3 The number of global points is the product of the numbers of local program points in all processes.
} 
has proposed the novel of concept of source sets, optimizing the implementation for DPOR. These methods, in general, often achieve better reduction than traditional techniques, due to the more accurate detection of independent transitions.

Traditional POR techniques $[20,18,9]$ distinguish between liveness and safety properties. POR has also been extended for symbolic model checking [2]: a symbolic state can represent a number of concrete states. These methods, however, are not applicable to safety verification of modern concurrent programs (written in mainstream APIs such as POSIX). One important weakness of traditional POR is that it is not sensitive wrt. different target safety properties. In contrast, recent works have shown that property-aware reduction can be achieved by symbolic methods using a general-purpose SAT/SMT solver $[24,14,22,5]$. Verification is often encoded as a formula which is satisfiable iff there exists an interleaving execution of the programs that violates the property. Reductions happen inside the SAT solver through the addition of learned clauses derived by conflict analysis [19]. This type of reduction is somewhat similar to what we call state interpolation.

An important related work is [14], which is the first to consider enhancing POR with property driven pruning, via the use of an SMT solver. Subsequently, there was a follow-up work [22]. In [14], they began with an SMT encoding of the underlying transition system, and then they enhance this encoding with a concept of "monotonicity". The effect of this is that traces can be grouped into equivalence classes, and in each class, all traces which are not monotonic will be considered as unsatisfiable by the SMT solver. The idea of course is that such traces are in fact redundant. This work has demonstrated some promising results as most concurrency bugs in real applications have been found to be shallow. We note that [14] incidentally enjoyed some (weak) form of SI pruning, due to the similarity between conflict clause learning and state interpolation. However, there the synergy between POR and SMT is unclear. We later demonstrate in Sec. 7 that such synergy in [14] is indeed relatively poor.

There is a fundamental problem with scalability in [14], as mentioned in the follow-up work [22]: "It will not scale to the entire concurrent program" if we encode the whole search space as a single formula and submit it to an SMT solver.

Let us first compare [14] with our work. Essentially, the difference is twofold. First, in this paper, the theory for partial order reduction is property driven. In contrast, the monotonicity reduction of [14] is not. In other words, though property driven pruning is observed in [14], it is contributed mainly by the conflict clauses learned, not from the monotonicity relation. We specifically exemplify the power of property driven POR in the later sections. Second, the encoding in [14] is processed by a black-box SMT solver. Thus important algorithmic refinements are not possible. Some examples:

- There are different options in implementing SI. Specifically in this paper, we employ "precondition" computations. Using a black-box solver, one has to rely on its fixed interpolation methods. 
- Our approach is lazy in a sense that our solver is only required to consider one symbolic path at a time; in [14] it is not the case. This matters most when the program is unsafe and finding counter-examples is relatively easy (there are many traces which violate the safety property).

- In having a symbolic execution framework, one can direct the search process. This is useful since the order in which state interpolants are generated does give rise to different reductions. Of course, such manipulation of the search process is hard, if not impossible, when using a black-box solver.

In order to remedy the scalability issue of [14], the work [22] adapted it to the setting of program testing. In particular, [22] proposed a concurrent trace program (CTP) framework which employs both concrete execution and symbolic solving to strike a balance between efficiency and scalability of an SMT-based method. However, when the input program is safe, i.e., absence of bugs, [22] in general suffers from the same scalability issue as in [14].

We remark that, the new direction of [22], in avoiding the blow-up of the SMT solver, was in fact preceded by the work on under-approximation widening (UW) [10]. As with CTP, UW models a subset, which will be incrementally enlarged, of all the possible interleavings as an SMT formula and submits it to an SMT solver. In UW the scheduling decisions are also encoded as constraints, so that the unsatisfiable core returned by the solver can then be used to further the search in probably a useful direction. This is the major contribution of UW. However, an important point is that this furthering of the search is a repeated call to the solver, this time with a weaker formula; which means that the problem at hand is now larger, having more traces to consider. On this repeated call, the work done for the original call is thus duplicated.

At first glance, it seems attractive and simple to encode the problem compactly as a set of constraints and delegate the search process to a general-purpose SMT solver. However, there are some fundamental disadvantages, and these arise mainly because it is hard to exploit the semantics of the program to direct the search inside the solver. This is in fact evidenced in the works mentioned above.

We believe, however, the foremost disadvantage of using a general-purpose solver lies in the encoding of process interleavings. For instance, even when a concurrent program has only one feasible execution trace, the encoding formula being fed to the solver is still of enormous size and can easily choke up the solver. More importantly, different from safety verification of sequential programs, the encoding of interleavings (e.g., [14] uses the variable sel to model which process is selected for executing) often hampers the normal derivations of succinct conflict clauses by means of resolution in modern SMT solvers. We empirically demonstrate the inefficiency of such approach in Sec. 7 .

Another important related work is [21], developed independently ${ }^{4}$ by Wachter et al. [21] follows a similar direction as in this paper: combining POR with a standard state interpolation algorithm, which is often referred to as the IMPACT

\footnotetext{
${ }^{4}$ Our work has been publicly available since 2012 in forms of a draft paper and a Ph.D. thesis. For anonymity reason, we omit the details here.
} 
algorithm [17]. Nevertheless, it is important to note that the framework presented in this paper subsumes [21]. While this paper proposes the novel concept of Property Driven POR before combining it with the state interpolation algorithm, [21] exploits directly the concept of "monotonicity" as in [14], thus their POR part does not give rise to property driven pruning.

\section{Background}

We consider a concurrent system composed of a finite number of threads or processes performing atomic operations on shared variables. Let $P_{i}(1 \leq i \leq n)$ be a process with the set trans $s_{i}$ of transitions. For simplicity, assume that trans $i$ contains no cycles.

We also assume all processes have disjoint sets of transitions. Let $\mathcal{T}=$ $\cup_{i=1}^{n}$ trans $_{i}$ be the set of all transitions. Let $V_{i}$ be the set of local variables of process $P_{i}$, and $V_{\text {shared }}$ the set of shared variables of the given concurrent program. Let $p c_{i} \in V_{i}$ be a special variable representing the process program counter, and the tuple $\left\langle p c_{1}, p c_{2} \cdots, p c_{n}\right\rangle$ represent the global program point. Let SymStates be the set of all global symbolic states of the given program where $s_{0} \in$ SymStates is the initial state. A state $s \in$ SymStates comprises two parts: its global program point $\ell$, also denoted by $\mathrm{pc}(s)$, which is a tuple of local program counters, and its symbolic constraints $\llbracket s \rrbracket$ over the program variables. In other words, we denote a state $s$ by $\langle\mathrm{pc}(s), \llbracket s \rrbracket\rangle$.

We consider the transitions of states induced by the program. Following [9], we only pay attention to visible transitions. A (visible) transition $t^{\{i\}}$ pertains to some process $P_{i}$. It transfers process $P_{i}$ from control location $\ell_{1}$ to $\ell_{2}$. In general, the application of $t^{\{i\}}$ is guarded by some condition cond (cond might be just true). At some state $s \in$ SymStates, when the $i^{\text {th }}$ component of $\mathrm{pc}(s)$, namely $\mathrm{pc}(s)[i]$, equals $\ell_{1}$, we say that $t^{\{i\}}$ is schedulable ${ }^{5}$ at $s$. And when $s$ satisfies the guard cond, denoted by $s=$ cond, we say that $t^{\{i\}}$ is enabled at $s$. For each state $s$, let Schedulable(s) and Enabled(s) denote the set of transitions which respectively are schedulable at $s$ and enabled at $s$. A state $s$, where Schedulable $(s)=\emptyset$, is called a terminal state.

Let $s \stackrel{t}{\rightarrow} s^{\prime}$ denote transition step from $s$ to $s^{\prime}$ via transition $t$. This step is possible only if $t$ is schedulable at $s$. We assume that the effect of applying an enabled transition $t$ on a state $s$ to arrive at state $s^{\prime}$ is well-understood. In our symbolic execution framework, executing a schedulable but not enabled transition results in an infeasible state. A state $s$ is called infeasible if $\llbracket s \rrbracket$ is unsatisfiable. For technical reasons needed below, we shall allow schedulable transitions emanating from an infeasible state; it follows that the destination state must also be infeasible.

For a sequence of transitions $w$ (i.e., $\left.w \in \mathcal{T}^{*}\right), \operatorname{Rng}(w)$ denotes the set of transitions that appear in $w$. Also let $\mathcal{T}_{\ell}$ denote the set of all transitions which are schedulable somewhere after global program point $\ell$. We note here that the

\footnotetext{
5 This concept is not standard in traditional POR, we need it here since we are dealing with symbolic search.
} 
schedulability of a transition at some state $s$ only depends on the program point component of $s$, namely $\mathrm{pc}(s)$. It does not depend on the constraint component of $\mathrm{s}$, namely $\llbracket s \rrbracket$. Given $t_{1}, t_{2} \in \mathcal{T}$ we say $t_{1}$ can de-schedule $t_{2}$ iff there exists a state $s$ such that both $t_{1}, t_{2}$ are schedulable at $s$ but $t_{2}$ is not schedulable after the execution of $t_{1}$ from $s$.

Following the above, $s_{1} \stackrel{t_{1} \cdots t_{m}}{=} s_{m+1}$ denotes a sequence of state transitions, and we say that $s_{m+1}$ is reachable from $s_{1}$. We call $s_{1} \stackrel{t_{1}}{\rightarrow} s_{2} \stackrel{t_{2}}{\rightarrow} \ldots \stackrel{t_{m}}{\rightarrow} s_{m+1}$ a feasible derivation from state $s_{1}$, iff $\forall 1 \leq i \leq m \bullet t_{i}$ is enabled at $s_{i}$. As mentioned earlier, an infeasible derivation results in an infeasible state (an infeasible state is still aware of its global program point). An infeasible state satisfies any safety property.

We define a complete execution trace, or simply trace, $\rho$ as a sequence of transitions such that it is a derivation from $s_{0}$ and $s_{0} \stackrel{\rho}{\Longrightarrow} s_{f}$ and $s_{f}$ is a terminal state. A trace is infeasible if it is an infeasible derivation from $s_{0}$. If a trace is infeasible, then at some point, it takes a transition which is schedulable but is not enabled. From thereon, the subsequent states are infeasible states.

We say a given concurrent program is safe wrt. a safety property $\psi$ if $\forall s \in$ SymStates $\bullet$ if $s$ is reachable from the initial state $s_{0}$ then $s=\psi$. A trace $\rho$ is safe wrt. $\psi$, denoted as $\rho \models \psi$, if all its states satisfy $\psi$.

\section{Partial Order Reduction (POR) vs. State-based Interpolation (SI)}

We assume the readers are familiar with the traditional concept of POR. Regarding state-based interpolation, we follow the approach of $[13,17]$. Here our symbolic execution is depicted as a tree rooted at the initial state $s_{0}$ and for each state $s_{i}$ therein, the descendants are just the states obtainable by extending $s_{i}$ with a feasible transition.

Definition 1 (Safe Root). Given a transition system and an initial state $s_{0}$, let $s$ be a feasible state reachable from $s_{0}$. We say $s$ is a safe root wrt. a safety property $\psi$, denoted $\Delta_{\psi}(s)$, iff all states $s^{\prime}$ reachable from s are safe wrt. $\psi$.

Definition 2 (State Coverage). Given a transition system and an initial state $s_{0}$ and $s_{i}$ and $s_{j}$ are two symbolic states such that (1) $s_{i}$ and $s_{j}$ are reachable from $s_{0}$ and (2) $s_{i}$ and $s_{j}$ share the same program point $\ell$, we say $s_{i}$ covers $s_{j}$ wrt. a safety property $\psi$, denoted by $s_{i} \succeq_{\psi} s_{j}$, iff $\Delta_{\psi}\left(s_{i}\right)$ implies $\Delta_{\psi}\left(s_{j}\right)$.

The impact of state coverage relation is that if (1) $s_{i}$ covers $s_{j}$, and (2) the subtree rooted at $s_{i}$ has been traversed and proved to be safe, then the traversal of subtree rooted at $s_{j}$ can be avoided. In other words, we gain performance by pruning the subtree at $s_{j}$. Obviously, if $s_{i}$ naturally subsumes $s_{j}$, i.e., $s_{j} \models s_{i}$, then state coverage is trivially achieved. In practice, however, this scenario does not happen often enough.

Definition 3 (Sound Interpolant). Given a transition system and an initial state $s_{0}$, given a safety property $\psi$ and program point $\ell$, we say a formula $\bar{\Psi}$ is a sound interpolant for $\ell$, denoted by $\mathrm{SI}(\ell, \psi)$, if for all states $s \equiv\langle\ell, \cdot\rangle$ reachable from $s_{0}, s \models \bar{\Psi}$ implies that $s$ is a safe root. 
What we want now is to generate a formula $\bar{\Psi}$ (called interpolant), which still preserves the safety of all states reachable from $s_{i}$, but is weaker (more general) than the original formula associated to the state $s_{i}$. In other words, we should have $s_{i} \models \mathrm{SI}(\ell, \psi)$. We assume that this condition is always ensured by any implementation of state-based interpolation. The main purpose of using $\bar{\Psi}$ rather than the original formula associated to the symbolic state $s_{i}$ is to increase the likelihood of subsumption. That is, the likelihood of having $s_{j} \models \bar{\Psi}$ is expected to be much higher than the likelihood of having $s_{j} \models s_{i}$.

In fact, the perfect interpolant should be the weakest precondition [7] computed for program point $\ell$ wrt. the transition system and the safety property $\psi$. We denote this weakest precondition as $\operatorname{wp}(\ell, \psi)$. Any subsequent state $s_{j} \equiv\langle\ell, \cdot\rangle$ which has $s_{j}$ stronger than this weakest precondition can be pruned. However, in general, the weakest precondition is too computationally demanding. An interpolant for the state $s_{i}$ is indeed a formula which approximates the weakest precondition at program point $\ell$ wrt. the transition system, i.e., $\bar{\Psi} \equiv \operatorname{SI}(\ell, \psi) \equiv \operatorname{Intp}\left(s_{i}, \mathrm{wp}(\ell, \psi)\right)$. A good interpolant is one which closely approximates the weakest precondition and can be computed efficiently.

The symbolic execution of a program can be augmented by annotating each program point with its corresponding interpolants such that the interpolants represent the sufficient conditions to preserve the unreachability of any unsafe state. Then, the basic notion of pruning with state interpolant can be defined as follows.

Definition 4 (Pruning with Interpolant). Given a symbolic state $s \equiv\langle\ell, \cdot\rangle$ such that $\ell$ is annotated with some interpolant $\bar{\Psi}$, we say that $s$ is pruned by the interpolant $\bar{\Psi}$ if s implies $\bar{\Psi}$ (i.e., $s \models \bar{\Psi}$ ).

Now let us discuss the the effectiveness of POR and SI in pruning the search space with an example. For simplicity, we purposely make the example concrete, i.e., states are indeed concrete states.

EXAMPle 1 (Closely coupled processes): See Fig. 1. Program points are shown in angle brackets. Fig. 1(a) shows the control flow graphs of two processes. Process 1 increments $x$ twice whereas process 2 doubles $x$ twice. The transitions associated with such actions and the safety property are depicted in the figure. POR requires a full search tree while Fig. 1(b) shows the search space explored by SI. Interpolants are in curly brackets. Bold circles denote pruned/subsumed states. Let us first attempt this example using POR. It is clear that $t_{1}^{\{1\}}$ is dependent with both $t_{1}^{\{2\}}$ and $t_{2}^{\{2\}}$. Also $t_{2}^{\{1\}}$ is dependent with both $t_{1}^{\{2\}}$ and $t_{2}^{\{2\}}$. Indeed, each of all the 6 execution traces in the search tree ends at a different concrete state. As classic POR methods use the concept of trace equivalence for pruning, no interleaving is avoided: those methods will enumerate the full search tree of 19 states (for space reasons, we omit it here).

Revisit the example using SI, where we use the weakest preconditions [7] as the state interpolants: the interpolant for a state is computed as the weakest precondition to ensure that the state itself as well as all of its descendants are safe (see Fig. 1(b)). We in fact achieve the best case scenario with it: whenever we 


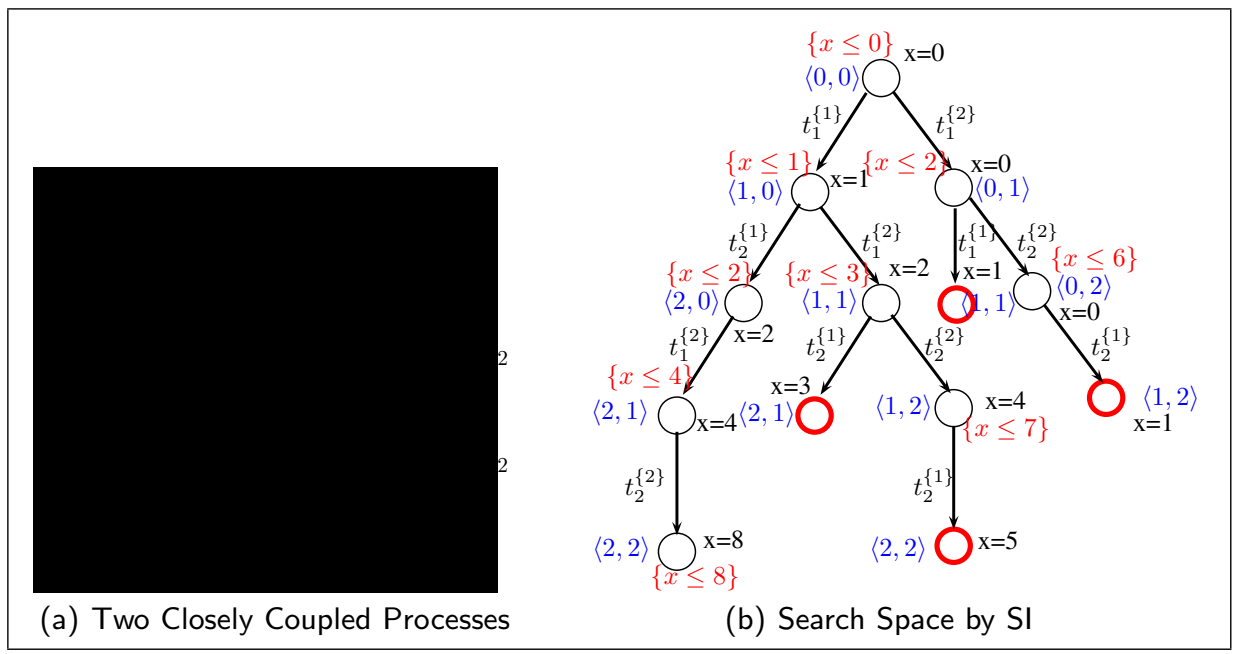

Fig. 1: Application of SI on 2 Closely Coupled Processes

come to a program point which has been examined before, subsumption happens. The number of non-subsumed states is still of order $O\left(k^{2}\right)$ (where $k=3$ in this particular example), assuming that we generalize the number of local program points for each process to $O(k)$. Fig. 1(b) shows 9 non-subsumed states and 4 subsumed states.

In summary, the above example shows that SI might outperform POR when the component processes are closely coupled. However, one can easily devise an example where the component processes do not interfere with each other at all. Under such condition POR will require only one trace to prove safety, while SI is still (lower) bounded by the total number of global program points. In this paper, we contribute by proposing a framework to combine POR and SI synergistically.

\section{Property Driven POR (PDPOR)}

"Combining classic POR methods with symbolic algorithms has been proven to be difficult" [14]. One fundamental reason is that the concepts of (Mazurkiewicz) equivalence and transition independence, which drive most practical POR implementations, rely on the equivalence of two concrete states. However, in symbolic traversal, we rarely encounter two equivalent symbolic states.

We now make the following definition which is crucial for the concept of pruning and will be used throughout this paper.

Definition 5 (Trace Coverage). Let $\rho_{1}, \rho_{2}$ be two traces of a concurrent program. We say $\rho_{1}$ covers $\rho_{2}$ wrt. a safety property $\psi$, denoted as $\rho_{1} \beth_{\psi} \rho_{2}$, iff $\rho_{1} \models \psi \rightarrow \rho_{2} \models \psi$.

Instead of using the concept of trace equivalence, from now on, we only make use of the concept of trace coverage. The concept of trace coverage is definitely weaker than the concept of Mazurkiewicz equivalence. In fact, if $\rho_{1}$ and $\rho_{2}$ are 
(Mazurkiewicz) equivalent then $\forall \psi \bullet \rho_{1} \sqsupseteq_{\psi} \rho_{2} \wedge \rho_{2} \sqsupseteq_{\psi} \rho_{1}$. Now we will define a new and weaker concept which therefore generalizes the concept of transition independence.

Definition 6 (Semi-Commutative After A State). For a given concurrent program, a safety property $\psi$, and a derivation $s_{0} \stackrel{\theta}{\Longrightarrow} s$, for all $t_{1}, t_{2} \in \mathcal{T}$ which cannot de-schedule each other, we say $t_{1}$ semi-commutes with $t_{2}$ after state $s$ wrt. $\beth_{\psi}$, denoted by $\left\langle s, t_{1} \uparrow t_{2}, \psi\right\rangle$, iff for all $w_{1}, w_{2} \in \mathcal{T}^{*}$ such that $\theta w_{1} t_{1} t_{2} w_{2}$ and $\theta w_{1} t_{2} t_{1} w_{2}$ are execution traces of the program, then we have $\theta w_{1} t_{1} t_{2} w_{2} \beth_{\psi} \theta w_{1} t_{2} t_{1} w_{2}$.

From the definition, $R n g(\theta), R n g\left(w_{1}\right)$, and $R n g\left(w_{2}\right)$ are pairwise disjoint. Importantly, if $s$ is at program point $\ell$, we have $R n g\left(w_{1}\right) \cup R n g\left(w_{2}\right) \subseteq \mathcal{T}_{\ell} \backslash\left\{t_{1}, t_{2}\right\}$. We observe that wrt. some $\psi$, if all important events, those have to do with the safety of the system, have already happened in the prefix $\theta$, the "semicommutative" relation is trivially satisfied. On the other hand, the remaining transitions might still interfere with each other (but not the safety), and do not satisfy the independent relation.

The concept of "semi-commutative" is obviously weaker than the concept of independence. If $t_{1}$ and $t_{2}$ are independent, it follows that $\forall \psi \forall s \bullet\left\langle s, t_{1} \uparrow t_{2}, \psi\right\rangle \wedge$ $\left\langle s, t_{2} \uparrow t_{1}, \psi\right\rangle$. Also note that, in contrast to the relation of transition independence, the "semi-commutative" relation is not symmetric.

We now introduce a new definition for persistent set.

Definition 7 (Persistent Set Of A State). A set $T \subseteq \mathcal{T}$ of transitions schedulable in a state $s \in$ SymStates is persistent in $s$ wrt. a property $\psi$ iff, for all derivations $s \stackrel{t_{1}}{\rightarrow} s_{1} \stackrel{t_{2}}{\rightarrow} s_{2} \ldots \stackrel{t_{m-1}}{\rightarrow} s_{m-1} \stackrel{t_{m}}{\rightarrow} s_{m}$ including only transitions $t_{i} \in \mathcal{T}$ and $t_{i} \notin T, 1 \leq i \leq m$, each transition in $T$ semi-commutes with $t_{i}$ after s wrt. $\beth_{\psi}$.

For each state, computing a persistent set from the "semicommutative" relation is similar to computing the classical persistent set under the transition independence relation. The algorithms for this task can be easily Fig. 2: Computing a Persistent Set of a State adapted from the algorithms presented in [9]. For convenience, we show one of such possibilities in Fig. 2.

As in traditional algorithms, the quality (i.e. the size) of the returned persistent set is highly dependent on the first transitioned $t$ to be added and the order in which the remaining transitions $t_{i}$ are considered. This is, however, not the topic of the current paper.

With the new definition of persistent set, we now can proceed with the normal selective search as described in classic POR techniques. In the algorithm presented in Fig. 3, we perform depth first search (DFS), and only accommodate safety verification (invariant property $\psi$ ). 
Theorem 1. The selective search algorithm in Fig. 3 is sound ${ }^{6}$.

In preparing for POR and SI to work together, we now further modify the concept of persistent set so that it applies for a set of states sharing the same program point. We remark that the previous definitions apply only for a specific state. The key intuition is to attach a precondition $\phi$ to the program point of interest, indicating when semi-

Safety property $\psi$ and initial state $s_{0}$

$\langle 1\rangle$ Initially : Explore $\left(s_{0}\right)$

function Explore $(s)$

$\langle 2\rangle$ if $s \not \neq \psi$ Report Error and TERMINATE

$\langle 3\rangle T:=$ Persistent_Set $(s)$

$\langle 4\rangle$ foreach enabled transition $t$ in $T$ do

$\langle 5\rangle \quad s \stackrel{t}{\longrightarrow} s^{\prime} \quad / *$ Execute $\mathrm{t}^{*} /$

$\langle 6\rangle \quad \operatorname{Explore}\left(s^{\prime}\right)$

end function

Fig. 3: New Selective Search Algorithm commutativity happens.

Definition 8 (Semi-Commutative After A Program Point). For a given concurrent program, a safety property $\psi$, and $t_{1}, t_{2} \in \mathcal{T}$, we say $t_{1}$ semi-commutes with $t_{2}$ after program point $\ell$ wrt. $\beth_{\psi}$ and $\phi$, denoted as $\left\langle\ell, \phi, t_{1} \uparrow t_{2}, \psi\right\rangle$, iff for all state $s \equiv\langle\ell, \cdot\rangle$ reachable from the initial state $s_{0}$, if $s=\phi$ then $t_{1}$ semicommutes with $t_{2}$ after state $s$ wrt. $\beth_{\psi}$.

Definition 9 (Persistent Set Of A Program Point). A set $T \subseteq \mathcal{T}$ of transitions schedulable at program point $\ell$ is persistent at $\ell$ under the interpolant $\bar{\Psi}$ wrt. a property $\psi$ iff, for all state $s \equiv\langle\ell, \cdot\rangle$ reachable from the initial state $s_{0}$, if $s=\bar{\Psi}$ then for all derivations $s \stackrel{t_{1}}{\rightarrow} s_{1} \stackrel{t_{2}}{\rightarrow} s_{2} \ldots \stackrel{t_{m-1}}{\rightarrow} s_{m-1} \stackrel{t_{m}}{\rightarrow} s_{m}$ including only transitions $t_{i} \in \mathcal{T}$ and $t_{i} \notin T, 1 \leq i \leq m$, each transition in $T$ semi-commutes with $t_{i}$ after state $s$ wrt. $\sqsupseteq_{\psi}$.

Assume that $T=\left\{t p_{1}, t p_{2}, \cdots t p_{k}\right\}$. The interpolant $\bar{\Psi}$ can now be computed as $\bar{\Psi}=\bigwedge \phi_{j i}$ for $1 \leq j \leq k, 1 \leq i \leq m$ such that $\left\langle\ell, \phi_{j i}, t p_{j} \uparrow t_{i}, \psi\right\rangle$.

For each program point, it is possible to have different persistent sets associated with different interpolants. In general, a state which satisfies a stronger interpolant will have a smaller persistent set, therefore, it enjoys more pruning.

\section{Synergy of PDPOR and SI}

We now show our combined framework. We assume for each program point, a persistent set and its associated interpolant are computed statically, i.e., by separate analyses. In other words, when we are at a program point, we can right away make use of the information about its persistent set.

The algorithm is in Fig. 4. The function Explore has input $s$ and assumes the safety property at hand is $\psi$. It naturally performs a depth first search of the state space.

\footnotetext{
${ }^{6}$ Proof outline is in the Appendix.
} 
Two Base Cases: The function Explore handles two base cases. One is when the current state is subsumed by some computed (and memoed) interpolant $\bar{\Psi}$. No further exploration is needed, and $\bar{\Psi}$ is returned as the interpolant (line 2). The second base case is when the current state is found to be unsafe (line 3).

Combining Interpolants: We make use of the (static) persistent set $T$ computed for the current program point. We comment further on this in the next section.

The set of transitions to be considered is denoted by Ts. When the current state implies the interpolant $\bar{\Psi}_{\text {trace }}$ associated with $T$, we need to consider only those transitions in $T$. Otherwise, we need to consider all the schedulable transitions. Note that when the persistent set $T$ is employed, the interpolant $\bar{\Psi}_{\text {trace }}$ must contribute to the combined interpolant of the current state (line 8). Disabled transitions at the current state will strengthen the interpolant as in line 11. Finally, we recursively follow those transitions which are enabled at the current state. The interpolant of each child state contributes to the interpolant of the current state as in line 15. In our framework, interpolants are propagated back using the precondition operation pre, where pre $(t, \phi)$ denotes a safe approximation of the weakest precondition wrt. the transition $t$ and the postcondition $\phi[7]$.

Theorem 2. The algorithm in Fig. 4 is sound $^{7}$.

\section{Implementation of PDPOR}

We now elaborate on the remaining task: how to estimate the semi-commutative relation, thus deriving the (static) persistent set at a program point. Similar to the formalism of traditional POR, our formalism is of paramount importance for the semantic use as well as to construct the formal proof of correctness (see the Appendix). In practice, however, we have to come up with sufficient conditions to efficiently implement the concepts. In this paper, we estimate the semi-commutative relation in two steps:

1. We first employ any traditional POR method and first estimate the "semicommutative" relation as the traditional independence relation (then the

\footnotetext{
7 Proof outline is in the Appendix.
} 
corresponding condition $\phi$ is just true). This is possible because the proposed concepts are strictly weaker than the corresponding concepts used in traditional POR methods.

2. We then identify and exploit a number of patterns under which we can statically derive and prove the semi-commutative relation between transitions. In fact, these simple patterns suffice to deal with a number of important real-life applications.

In the rest of this section, we outline three common classes of problems, from which the semi-commutative relation between transitions can be easily identified and proved, i.e., our step 2 becomes applicable.

Resource Usage of Concurrent Programs: Programs make use of limited resource (such as time, memory, bandwidth). Validation of resource usage in sequential setting is already a hard problem. It is obviously more challenging in the setting of concurrent programs due to process interleavings.

Here we model this class of problems by using a resource variable r. Initially, $r$ is zero. Each process can increment or decrement variable $r$ by some concrete value (e.g., memory allocation or deallocation respectively). A process can also double the value $r$ (e.g., the whole memory is duplicated). However, the resource variable $r$ cannot be used in the guard condition of any transition, i.e., we cannot model the behavior of a typical garbage collector. The property to be verified is that, "at all times, $r$ is (upper-) bounded by some constant".

Proposition 1. Let $r$ be a resource variable of a concurrent program, and assume the safety property at hand is $\psi \equiv r \leq C$, where $C$ is a constant. For all transitions (assignment operations only) $t_{1}: r=r+c_{1}, t_{2}: r=r * 2, t_{3}: r=r-c_{2}$ where $c_{1}, c_{2}>0$, we have for all program points $\ell$ :

$\left\langle\ell\right.$, true $\left._{1} t_{1} \uparrow t_{2}, \psi\right\rangle \wedge\left\langle\ell\right.$, true $\left., t_{1} \uparrow t_{3}, \psi\right\rangle \wedge\left\langle\ell\right.$, true $\left., t_{2} \uparrow t_{3}, \psi\right\rangle$

Informally, other than common mathematical facts such as additions can commute and so do multiplications and subtractions, we also deduce that additions can semi-commute with both multiplications and subtractions while multiplications can semi-commute with subtractions. This Proposition can be proved by using basic laws of algebra.

EXAMPLE 2 : Let us refer back to the example of two closely coupled processes introduced in Sec. 3, but now under the assumption that $x$ is the resource variable of interest. Using the semi-commutative relation derived from Proposition 1, we need to explore only one complete trace to prove this safety.

We recall that, in contrast, POR (and DPOR)-only methods will enumerate the full execution tree which contains 19 states and 6 complete execution traces. Any technique which employs only the notion of Mazurkiewicz trace equivalence for pruning will have to consider all 6 complete traces (due to 6 different terminal states). SI alone can reduce the search space in this example, and requires to explore only 9 states and 4 subsumed states (as in Sec. 3).

Detection of Race Conditions: [23] proposed a property driven pruning algorithm to detect race conditions in multithreaded programs. This work has achieved more reduction in comparison with DPOR. The key observation is that, 
at a certain location (program point) $\ell$, if their conservative "lockset analysis" shows that a search subspace is race-free, the subspace can be pruned away. As we know, DPOR relies solely on the independence relation to prune redundant interleavings (if $t_{1}, t_{2}$ are independent, there is no need to flip their execution order). In [23], however, even when $t_{1}, t_{2}$ are dependent, we may skip the corresponding search space if flipping the order of $t_{1}, t_{2}$ does not affect the reachability of any race condition. In other words, [23] is indeed a (conservative) realization of our PDPOR, specifically targeted for detection of race conditions. Their mechanism to capture the such scenarios is by introducing a trace-based lockset analysis.

Ensuring Optimistic Concurrency: In the implementations of many concurrent protocols, optimistic concurrency, i.e., at least one process commits, is usually desirable. This can be modeled by introducing a flag variable which will be set when some process commits. The flag variable once set can not be unset. It is then easy to see that for all program point $\ell$ and transitions $t_{1}, t_{2}$, we have $\left\langle\ell\right.$, flag $\left.=1, t_{1} \uparrow t_{2}, \psi\right\rangle$. Though simple, this observation will bring us more reduction compared to traditional POR methods.

\section{$7 \quad$ Experiments}

This section conveys two key messages. First, when trace-based and state-based methods are not effective individually, our combined framework still offers significant reduction. Second, property driven POR can be very effective, and applicable not only to academic programs, but also to programs used as benchmarks in the state-of-the-art.

We use a 3.2 GHz Intel processor and 2GB memory running Linux. Timeout is set at 10 minutes. In the tables, cells with '-' indicate timeout. We compare the performance of Partial Order Reduction alone (POR), State Interpolation alone (SI), the synergy of Partial Order Reduction and State Interpolation (POR+SI), i.e., the semi-commutative relation is estimated using only step 1 presented in Sec. 6, and when applicable, the synergy of Property Driven Partial Order Reduction and State Interpolation (PDPOR+SI), i.e., the semi-commutative relation is estimated using both steps presented in Sec. 6. For the POR component, we use the implementation from [3].

Table 1 starts with parameterized versions of the producer/consumer example because its basic structure is extremely common. There are $2 * N$ producers and 1 consumer. Each producer will do its own non-interfered computation first, modeled by a transition which does not interfere with other processes. Then these producers will modify the shared variable $x$ as follows: each of the first $N$ producers increments $x$, while the other $N$ producers double the value of $x$. On the other hand, the consumer consumes the value of $x$. The safety property is that the consumed value is no more than $N * 2^{N}$.

Table 1 clearly demonstrates the synergy benefits of POR and SI. POR+SI significantly outperforms both POR and SI. Note that this example can easily be translated to the resource usage problem, where our PDPOR requires only a single trace (and less than 0.01 second) in order to prove safety. 
We next use the parameterized version of the dining philosophers. We chose this for two reasons. First, this is a classic example often used in concurrent algorithm design to illustrate synchronization issues and techniques for resolving them. Second, previous work [14] has used this to demonstrate benefits from combining POR and SMT.

The first safety property used in [14], "it is not that all philosophers can eat simultaneously", is somewhat trivial. Therefore, here we verify a tight property, which is (a): "no more than half the philosophers can eat simultaneously". To demonstrate the power of symbolic execution, we verify this property without knowing the initial configurations of all the forks. Table 1 , again, demonstrates the significant improvements of POR+SI over POR alone and $\mathrm{SI}$ alone. We note that the performance of our $\mathrm{POR}+\mathrm{SI}$ algorithm is about 3 times faster than [14].

We additionally considered a second safety property as in [14], namely (b): "it is possible to reach a state in which all philosophers have eaten at least once". Our symbolic execution framework requires only a single trace (and less than 0.01 second) to prove this property in all instances, whereas [14] requires even more time compared to proving property (a). This illustrates the scalability issue of [14], which is representative for other techniques employing general-purpose SMT solver for symbolic pruning.

We also perform experiments on the "Bakery" algorithm. Due to existence of infinite domain variables, model checking hardly can handle this case. Here we remark that in symbolic methods, loop handling is often considered as an orthogonal issue. Programs with statically bounded loops can be easily unrolled into equivalent loop-free programs. For unbounded loops, either loop invariants are provided or the employment of some invariant discovery routines, e.g., [12], is necessary. In order for our algorithm to work here, we make use of the standard loop invariant for this example.

To further demonstrate the power our synergy framework over $[14]^{8}$ as well as the power of our property driven POR, we experiment next on the Sumof-ids program. Here, each process (of $N$ processes) has one unique $i d$ and will

\footnotetext{
8 [14] is not publicly available. Therefore, it is not possible for us to make more comprehensive comparisons.
} 
increment a shared variable sum by this $i d$. We prove that in the end this variable will be incremented by the sum of all the ids.

See Table 2, where we experiment with Z3 [6] (version 4.1.2) using the encodings presented in [14]. \#C denotes the number of conflicts while \#D denotes the number of decisions made by Z3. We can see that our synergy Table 2: Comparison with [14]

\begin{tabular}{|c|c|c|c|c|c|c|}
\hline & \multicolumn{3}{|c|}{ [14] w. Z3 } & POR+SI & PDPOF & $\mathrm{R}+\mathrm{SI}$ \\
\hline$T(s)$ & $\# \mathrm{C}$ & \#D & $\mathrm{T}(\mathrm{s})$ & $\begin{array}{l}\text { States } \mathrm{T}(\mathrm{s}) \\
\end{array}$ & States & $\mathrm{T}(\mathrm{s})$ \\
\hline um-6 & 1608 & 1795 & 0.08 & \begin{tabular}{l|l|l|}
193 & 0.05 \\
\end{tabular} & 7 & 10 \\
\hline sum-8 & 54512 & 59267 & 10.88 & \begin{tabular}{l|l}
1025 & 0.27
\end{tabular} & 9 & 0.01 \\
\hline $\mathrm{m}-10$ & - & - & - & \begin{tabular}{ll|l}
5121 & 1.52
\end{tabular} & 11 & \\
\hline$u m-12$ & - & - & - & \begin{tabular}{l|l|l}
24577 & 8.80
\end{tabular} & 13 & 0. \\
\hline jum-14 & - & - & & $\mid 11468967.7$ & 15 & .0 \\
\hline
\end{tabular}
framework scale much better than [14] with Z3. Also, this example can also be translated to resource usage problem, our use of property-driven POR again requires one single trace to prove safety.

Finally, to benchmark our framework with SMT-based methods, we select four safe programs from [5] where the experimented methods did not perform well. Those programs are micro_2, stack, circular_buffer, and stateTable 3: Experiments on [5]'s Programs

\begin{tabular}{|l|r|r||r|r||r|r|}
\hline & \multicolumn{1}{|c|}{$[5]$} & \multicolumn{2}{|c|}{ SI } & \multicolumn{2}{|c|}{ PDPOR+SI } \\
\hline Problem & C & T(s) & States & T(s) & States & T(s) \\
\hline micro_2 & 17 & 1095 & 20201 & 10.88 & 201 & 0.04 \\
stack & 12 & 225 & 529 & 0.26 & 529 & 0.26 \\
circular_buffer & $\infty$ & 477 & 29 & 0.03 & 29 & 0.03 \\
stateful20 & 10 & 95 & 1681 & 1.13 & 41 & 0.01 \\
\hline
\end{tabular}
ful20. We note that safe programs allow fairer comparison between different approaches since to verify them we have to cover the whole search space. Table 3 shows the running time of $\mathrm{SI}$ alone and of the combined framework. For convenience, we also tabulate the best running time reported in [5] and $\mathrm{C}$ is the context switch bound used. We assume no context switch bound, hence the corresponding value in our framework is $\infty$.

We can see that even our SI alone significantly outperforms the techniques in [5]. We believe it is due to the inefficient encoding of process interleavings (mentioned in Sec. 2) as well as the following reasons. First, our method is lazy, which means that only a path is considered at a time: [5] itself demonstrates partially the usefulness of this. Second, but importantly, we are eager in discovering infeasible paths. The program circular_buffer, which has only one feasible complete execution trace, can be efficiently handled by our framework, but not SMT. This is one important advantage of our symbolic execution framework over SMT-based methods, as discussed in [17].

It is important to note that, PDPOR significantly improves the performance of SI wrt. programs micro_2 and stateful20. This further demonstrates the applicability of our proposed framework.

\section{Conclusion}

We present a concurrent verification framework which synergistically combines trace-based reduction techniques with the recently established notion of state interpolant. One key contribution is the new concept of property-driven POR which serves to reduce more interleavings than previously possible. We believe 
that fully automated techniques to compute the semi-commutative relations for specific, but important, application domains will be interesting future work.

\section{References}

1. P. Abdulla, S. Aronis, B. Jonsson, and K. Sagonas. Optimal dynamic partial order reduction. In POPL, 2014.

2. R. Alur, R. K. Brayton, T. A. Henzinger, S. Qadeer, and S. K. Rajamani. Partialorder reduction in symbolic state space exploration. In $C A V, 1997$.

3. P. Bokor, J. Kinder, M. Serafini, and N. Suri. Supporting domain-specific state space reductions through local partial-order reduction. In ASE, 2011.

4. C. Cadar, P. Godefroid, S. Khurshid, C. S. Păsăreanu, K. Sen, N. Tillmann, and W. Visser. Symbolic execution for software testing in practice: preliminary assessment. In ICSE, 2011.

5. L. Cordeiro and B. Fischer. Verifying multi-threaded software using smt-based context-bounded model checking. In ICSE, 2011.

6. L. De Moura and N. Bjørner. Z3: an efficient smt solver. In TACAS, 2008.

7. E. W. Dijkstra. Guarded commands, nondeterminacy and formal derivation of programs. Commun. ACM, 1975.

8. C. Flanagan and P. Godefroid. Dynamic partial-order reduction for model checking software. In POPL, 2005.

9. P. Godefroid. Partial-Order Methods for the Verification of Concurrent Systems: An Approach to the State-Explosion Problem. Springer-Verlag New York, Inc., 1996.

10. O. Grumberg, F. Lerda, O. Strichman, and M. Theobald. Proof-guided underapproximation-widening for multi-process systems. In POPL, 2005.

11. G. Gueta, C. Flanagan, E. Yahav, and M. Sagiv. Cartesian partial-order reduction. In SPIN, 2007.

12. J. Jaffar, J.A. Navas, and A. Santosa. Unbounded Symbolic Execution for Program Verification. In $R V, 2011$.

13. J. Jaffar, A. E. Santosa, and R. Voicu. An interpolation method for clp traversal. In $C P, 2009$.

14. V. Kahlon, C. Wang, and A. Gupta. Monotonic partial order reduction: An optimal symbolic partial order reduction technique. In $C A V, 2009$.

15. J. C. King. Symbolic Execution and Program Testing. Com. ACM, 1976.

16. A. W. Mazurkiewicz. Trace theory. In Advances in Petri Nets, 1986.

17. K. L. McMillan. Lazy annotation for program testing and verification. In $C A V$, 2010.

18. D. Peled. All from one, one for all: on model checking using representatives. In $C A V, 1993$.

19. J. P. M. Silva and K. A. Sakallah. Grasp-a new search algorithm for satisfiability. In $I C C A D, 1996$.

20. A. Valmari. Stubborn sets for reduced state space generation. In Applications and Theory of Petri Nets, 1989.

21. B. Wachter, D. Kroening, and J. Ouaknine. Verifying multi-threaded software with impact. In $F M C A D, 2013$.

22. C. Wang, S. Chaudhuri, A. Gupta, and Y. Yang. Symbolic pruning of concurrent program executions. In ESEC/FSE, 2009.

23. C. Wang, Y. Yang, A. Gupta, and G. Gopalakrishnan. Dynamic model checking with property driven pruning to detect race conditions. In ATVA, 2008.

24. C. Wang, Z. Yang, V. Kahlon, and A. Gupta. Peephole partial order reduction. In TACAS, 2008.

25. Y. Yang, X. Chen, G. Gopalakrishnan, and R. M. Kirby. Efficient stateful dynamic partial order reduction. In SPIN, 2008. 


\section{Appendix: Proofs of the Two Theorems}

Theorem 1. The selective search algorithm in Fig. 3 is sound.

Proof (Outline). Assume that there exist some traces which violate the property $\psi$ and are not examined by our selective search. Let denote the set of such traces as $\mathcal{W}_{\text {violated }}$. For each trace $\rho=s_{0} \stackrel{t_{1}}{\rightarrow} s_{1} \stackrel{t_{2}}{\rightarrow} s_{2} \cdots \stackrel{t_{m}}{\rightarrow} s_{m}, \rho \in \mathcal{W}_{\text {violated }}$, let first $(\rho)$ denote the smallest index $i$ such that $t_{i}$ is not in the persistent set of $s_{i-1}$. Without loss of generality, assume $\rho_{\max }=s_{0} \stackrel{t_{1}}{\rightarrow} s_{1} \stackrel{t_{2}}{\rightarrow} s_{2} \ldots \stackrel{t_{m}}{\rightarrow} s_{m}$ having the maximum $\operatorname{first}(\rho)$. Let $i=\operatorname{first}\left(\rho_{\max }\right)<m$. As the "commuter" and "commutee" cannot "de-schedule" each other, in the set $\left\{t_{i+1} \cdots t_{m}\right\}$ there must be a transition which belongs to the persistent set of $s_{i-1}$ (otherwise, the must exist some transition that belongs to the persistent set of $s_{i-1}$ which is schedulable at $s_{m}$. Therefore $s_{m}$ is not a terminal state). Let $j$ be the smallest index such that $t_{j}$ belongs to the persistent set of $s_{i-1}$. By definition, wrt. $\sqsupseteq_{\psi}$ and after $s_{i-1}, t_{j}$ semi-commutes with $t_{i}, t_{i+1}, \cdots t_{j-1}$. Also due to the definition of the "semi-commutative" relation we deduce that all the following traces (by making $t_{j}$ repeatedly commute backward):

$$
\begin{aligned}
& \rho_{1}^{\prime}=t_{1} t_{2} \cdots t_{i-1} t_{i} t_{i+1} \cdots t_{j} t_{j-1} t_{j+1} \cdots t_{m} \\
& \vdots \\
& \rho_{j-i-1}^{\prime}=t_{1} t_{2} \cdots t_{i-1} t_{i} t_{j} t_{i+1} \cdots t_{j-1} t_{j+1} \cdots t_{m} \\
& \rho_{j-i}^{\prime}=t_{1} t_{2} \cdots t_{i-1} t_{j} t_{i} t_{i+1} \cdots t_{j-1} t_{j+1} \cdots t_{m}
\end{aligned}
$$

must violate the property $\psi$ too. However, $\operatorname{first}\left(\rho_{j-i}^{\prime}\right)>\operatorname{first}\left(\rho_{\max }\right)$. This contradicts the definition of $\rho_{\max }$.

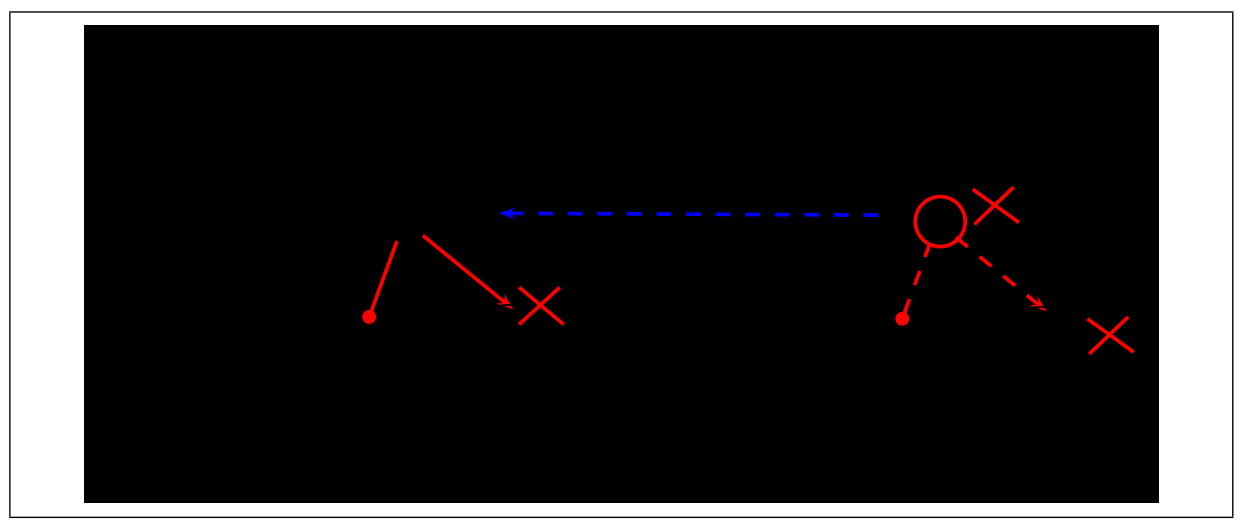

Fig. 5: Inductive Correctness

Theorem 2. The synergy algorithm in Fig. 4 is sound.

Proof (Outline). We use structural induction. Refer to Fig. 5. Assume that from $s_{0}$ we reach state $s_{i} \equiv\langle\ell, \cdot\rangle$. W.l.o.g., assume that at $s_{i}$ there are three transitions which are schedulable, namely $t_{1}, t_{2}, t_{3}$, of which only $t_{1}$ and $t_{2}$ are enabled. Also assume that under the interpolant $\bar{\Psi}_{2}$, the persistent set of $\ell$, and therefore of 
$s_{i}$, is just $\left\{t_{1}\right\}$. From the algorithm, we will extend $s_{i}$ with $t_{1}$ (line 13) and attempt to verify the subtree $A$ (line 14). Our induction hypothesis is that we have finished considering $A$, and indeed, it is safe under the interpolant $\bar{\Psi}_{A}$. That subtree will contribute $\bar{\Psi}_{1}=\operatorname{pre}\left(t_{1}, \bar{\Psi}_{A}\right)$ (line 15) to the interpolant of $s_{i}$.

Using the interpolant $\bar{\Psi}_{2}$, the branch having transition $t_{2}$ followed by the subtree $B$ is pruned and that is safe, due to Theorem 1 . Also, the disabled transition $t_{3}$ contributes $\bar{\Psi}_{3}$ (line 11) to the interpolant of $s_{i}$. Now we need to prove that $\bar{\Psi}=\bar{\Psi}_{1} \wedge \bar{\Psi}_{2} \wedge \bar{\Psi}_{3}$ is indeed a sound interpolant for program point $\ell$.

Assume that subsequently in the search, we reach some state $s_{j} \equiv\langle\ell, \cdot\rangle$. We will prove that $\bar{\Psi}$ is a sound interpolant of $\ell$ by proving that if $s_{j} \models \bar{\Psi}$, then the pruning of $s_{j}$ is safe.

First, $s_{j} \models \bar{\Psi}$ implies that $s_{j} \models \bar{\Psi}_{3}$. Therefore, at $s_{j}, t_{3}$ is also disabled. Second, assume that $t_{1}$ is enabled at $s_{j}$ and $s_{j} \stackrel{t_{1}}{\longrightarrow} s_{j+1}$ (if not the pruning of $t_{1}$ followed by $A^{\prime}$ is definitely safe). Similarly, $s_{j} \models \bar{\Psi}$ implies that $s_{j} \models \bar{\Psi}_{1}$. Consequently, $s_{j+1} \models \bar{\Psi}_{A}$ and therefore the subtree $A^{\prime}$ is safe too. Lastly, $s_{j} \models \bar{\Psi}$ implies that $s_{j}=\bar{\Psi}_{2}$. Thus the reasons which ensure that the traces ending with subtree $A$ cover the traces ending with subtree $B$ also hold at $s_{j}$. That is, the traces ending with subtree $A^{\prime}$ also cover the traces ending with subtree $B^{\prime}$. 